\title{
Treatment strategies of ruptured posterior inferior cerebellar artery aneurysm according to its segment
}

\author{
Jihye Song, Jung Eon Park ${ }^{1}$, Joonho Chung ${ }^{2}$, Yong Chul Lim³ , Yong Sam Shin ${ }^{4}$ \\ Department of Neurosurgery, Konyang College of Medicine, Konyang University Hospital, Daejeon, 'Department of Neurosurgery, Hankook Hospital, \\ Cheongju, ${ }^{2}$ Department of Neurosurgery, Gangnam Severance Hospital, Yonsei University College of Medicine, ${ }^{4}$ Department of Neurosurgery, \\ College of Medicine, Seoul St. Mary's Hospital, The Catholic University of Korea, Seoul, ${ }^{3}$ Department of Neurosurgery, The Ajou University School of Medicine, \\ Ajou University Hospital, Suwon, Republic of Korea \\ E-mail: Jihye Song - songjihye81@gmail.com; Jung Eon Park - jungeori98@hanmail.net; Joonho Chung - ns.joonho.chung@gmail.com; \\ Yong Chul Lim - nsyclim@gmail.com;*Yong Sam Shin - nsshin@gmail.com \\ *Corresponding author
}

Received: 03 April $16 \quad$ Accepted: 17 March $17 \quad$ Published: 25 July 17

\begin{abstract}
Background: The aim of this study was to evaluate the clinical outcomes between endovascular treatment (EVT) and surgery of the ruptured posterior inferior cerebellar artery (PICA) aneurysms and suggest proper management strategies according to the PICA segment.

Methods: We retrospectively analyzed clinical and radiological data of patients with ruptured PICA aneurysms who underwent surgery or EVT from three different institutes. Clinical outcomes were evaluated by $\mathrm{mRS}$.

Results: We collected 39 consecutive patients with 39 ruptured PICA aneurysms; 26 patients were with proximal segment aneurysm and 13 patients were with distal. Of the 26 proximal PICA aneurysms, 20 aneurysms were treated with EVT and 6 aneurysms were treated with surgery. EVT seemed to have more favorable clinical outcome ( $\mathrm{mRS} 0-2$; mean $\mathrm{mRS}, 1.75$ for EVT vs 3.50 for surgery, $P=0.152$ ). Of the 13 distal PICA aneurysms, 7 aneurysms were treated with EVT and 6 were treated with surgery. Surgery showed more favorable clinical outcome (mean mRS, 2.63 for EVT vs 0.33 for surgery, $P=0.023$ ) and lower procedure-related complication rate $(42.9 \%$ for EVT vs $0 \%$ for surgery, $P=0.046)$ than EVT.

Conclusions: For the treatment of ruptured PICA aneurysms, EVT seemed to have more favorable outcomes for proximal segment, while surgery showed more favorable outcomes for distal segment. Additional experience and follow-up are needed in a larger series to state the efficacy of this strategy.
\end{abstract}

Key Words: Endovascular treatment, intracranial aneurysm, posterior inferior cerebellar aneurysm, surgery

Access this article online Website:

www.surgicalneurologyint.com DOI:

10.4103/sni.sni_132_16

Quick Response Code:

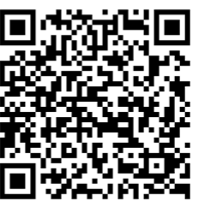

This is an open access article distributed under the terms of the Creative Commons Attribution-NonCommercial-ShareAlike 3.0 License, which allows others to remix, tweak, and build upon the work non-commercially, as long as the author is credited and the new creations are licensed under the identical terms.

For reprints contact: reprints@medknow.com

How to cite this article: Song J, Park JE, Chung J, Lim YC, Shin YS. Treatment strategies of ruptured posterior inferior cerebellar artery aneurysm according to its segment. Surg Neurol Int 2017;8:155.

http://surgicalneurologyint.com/Treatment-strategies-of-ruptured-posterior-inferior-cerebellar-artery-aneurysm-according-to-its-segment/ 


\section{INTRODUCTION}

The posterior inferior cerebellar artery (PICA) is very tortuous, has a small diameter, and projects along the brainstem and cerebellum. ${ }^{[4]}$ Critical perforators supplying the medulla originate from the proximal segment of the PICA, and aneurysms at these sites are at a high risk of rebleeding due to the thin wall. ${ }^{[4]}$ The incidence of lower cranial nerve palsy (LCNP) while manipulating the PICA is high in patients with a proximal PICA aneurysm through lateral and far-lateral suboccipital approaches. ${ }^{[1,4,12]}$ And the narrow and tortuous characteristics of the PICA during endovascular treatment (EVT) can cause vessel dissection, perforation, vasospasm, or aneurysmal rupture. Additionally, asymptomatic or symptomatic embolic infarct and unexpected parent artery occlusion can occur during EVT. Hence, it is often challenging to treat PICA aneurysm by both endovascular and microsurgical treatments. Before deciding on a management strategy, neurosurgeons should consider various predisposing factors, such as the initial clinical status of the patient, the location of the aneurysm, and the relationship between the aneurysm and parent artery, in order to achieve the best results and avoid complications. To the best of our knowledge, there are no previous reports comparing clinical results after EVT and surgery based on the involved segment. We retrospectively analyzed and compared the overall results of the patients with ruptured PICA aneurysms between EVT and surgery according to the involved PICA segment (proximal and distal).

\section{MATERIALS AND METHODS}

This study was approved by our institutional review board. The requirement to obtain written informed consent to participate in this study was waived. We retrospectively collected data of patients who were treated for ruptured PICA aneurysms in three referral hospitals (Seoul St. Mary's Hospital, Ajou University Hospital and Severance Hospital). Those three institutions have a tertiary neurointerventional center, and their neurointerventionists completed a fellowship program with Dr. YS Shin, therefore, the neurointerventionists were expected to follow similar management strategies for aneurysm treatment. From October 2002 to April 2013, 39 patients with 39 cases of PICA aneurysm were included (mean age 58.9 years; range). One of them had a failed endovascular treatment, after which microsurgical treatment was performed).

There were 7 men and 32 women (mean age 58.9 years, range: 21-75 years). The mean follow-up duration was 19 months (range: 1 month -6 years). The PICA infarct area was confirmed by a post-procedure CT after surgery or by diffusion MRI after EVT and neurologic changes before and after treatment. All except 10 patients (three patients lost follow-up, and seven patients died) were evaluated by MR angiography, CT angiography, or transfemoral cerebral angiography (TFCA) after treatment.

The PICA was divided into 5 segments according to the classification by Lister et al. ${ }^{[8]}$ Proximal PICA was defined as the area from the vertebral artery - PICA junction to the tonsillomedullar segment, which existed with brainstem perforators. All others were classified as distal PICA. Angiographic occlusion grade was determined by modified Raymond scale into complete occlusion, neck remnant, and incomplete occlusion. Clinical outcome was described using mRS and the mean mRS except medical mortality who died of medical cause, such as miocardiac infarct, sepsis, or acute respiratory distress syndrome, was also evaluated. Favorable outcome was defined as mRS 0-2. Clinical characteristics, radiologic findings, surgical procedure, intraoperative findings, and treatment outcome were extracted from medical records and neuroimaging data.

All patients with ruptured aneurysms were treated within 24 hours. Endovascular treatment was performed under general anesthesia. If a stent needed to be implanted, $200 \mathrm{mg}$ of aspirin and $300 \mathrm{mg}$ of clopidogrel were loaded through the Levin-tube in patients. These patients then maintained on daily doses of $100 \mathrm{mg}$ of aspirin and $75 \mathrm{mg}$ of clopidogrel for 3 to 6 months, followed by aspirin $100 \mathrm{mg}$ daily for 12 months.

The choice of the treatment strategy was made through multidisciplinary team. Selective endosaccular coiling was performed in the case of reasonable neck morphology, and if vessels were tortuous or had a narrow lumen (not wide enough to insert the microcatheter) and suspected of a distal PICA dissection with well-developed collateral circulations, parent artery occlusion, or ligation was considered. For microsurgical treatments, lateral suboccipital craniotomy was considered in patients with proximal PICA aneurysms, and midline suboccipital craniotomy in patients with distal PICA aneurysms.

SPSS version 18 was used for statistical analyses (SPSS Inc., Chicago, Illinois, USA). Statistical analyses were performed by use of $t$-tests and one-way analyses of variance (ANOVAs) for continuous variables, and $\chi^{2}$ or Fisher's exact test for categorical variables. Differences were considered statistically significant if $P$ values were less than 0.05 .

\section{RESULTS}

Arteries of the 39 patients were ruptured on admission. Twenty-six patients had proximal PICA aneurysms. Vertebral artery (VA) - PICA junction in 25 cases, anterior medullary segment in one case, while 13 aneurysms were located at distal segments. 
Clinical and radiological results and patient data for included PICA aneurysms are described according to the treatment modalities [Table 1]. There was no statistically significant difference of age, sex, or initial Hunt-Hess grade $(\mathrm{HH})$ between the EVT and surgery group. All EVTs were successful. There was no rebleeding during the follow-up period after treatment (mean, 17 months; range; $0.9-72$ months). Three patients $(15 \%, 3 / 39)$ showed recurrence after treatment. One patient was follow-up loss. Another patient was performed stent-assisted coil embolization two years later, and the other had undergone surgery after one year. All patients had showed complete occlusion of aneurysm on follow-up images except for the 7 patients who died and 3 who were lost-to-follow-up.

Table 2 summarizes the treatment-related complications, their clinical outcomes, and mortality cases. Of the 26 patients with proximal PICA aneurysm, 20 were treated with EVT (only two patients received stent-assisted coil embolization) and 6 were treated with aneurysm clipping. Among the EVT group, $12(60 \%, 12 / 20)$ aneurysms showed complete occlusion, three had partial, and five had incomplete occlusions. There were three cases of mortality that died of medical cause (cases 6, 7, and 8) among EVT group and one case of those (case 11) among surgery group. The mean mRS of EVT group was 1.75. In 6 patients who were treated with surgery, there were no recurrence and attempted failures. Surgery-related medullary infarct was found in two patients $(33.3 \%, 2 / 6$, cases 9 and 10$)$. The mean mRS of surgery group was 3.50 . EVT seemed to have a more favorable clinical outcome for the treatment of proximal aneurysm according to mean mRS (1.75 for EVT vs 3.50 for surgery, $P=0.152$ ).

Of the 13 patients with distal PICA aneurysm, 7 patients were treated by EVT (four of them performed parent artery occlusion using detachable coils or N-Butyl Cyanoacrylate glue), and 6 patients were treated by surgery (two of them were performed via trapping without anastomosis). There was no mortality by medical cause. In the EVT group, all aneurysms were completely occluded and had no recurrence. Four patients $(57.1 \%, 4 / 7)$ showed a favorable outcome and the mean mRS was 2.63. In the surgical group, there were no treatment failures and recurrence. The treatment-related complication rate and the mean mRS of surgery was significantly lower than those of EVT (0\% vs $42.9 \%, P=0.046 ; 2.63$ vs 0.33 , $P=0.023$.

\section{DISCUSSION}

PICA aneurysm is very rare, making up approximately $0.49-3.0 \%$ of all intracranial aneurysms. ${ }^{[4,5]}$ Almost all patients with ruptured PICA aneurysms visit to the emergency room with a poor initial neurological status. Because the PICA is located near the fourth ventricle, over $83 \%$ of patients with a ruptured aneurysm (Kallmes et al. reported 95\%) showed intraventricular hemorrhage (IVH) and obstructive hydrocephalus because of blood entering either through the foramen of Luschka or Magendie. ${ }^{[13]}$ Rebleeding rates were also high (about 78\%) due to the relatively thin aneurysm wall and its dissecting nature. ${ }^{[2]}$ In these cases, an external ventricular drainage catheter was inserted to control the increased intracranial pressure, such as IVH or obstructive hydrocephalus, and then urgent microsurgical or endovascular treatment was recommended. Such proper and urgent management strategies resulted in over $76.8 \%$ excellent outcomes in most of the reports. ${ }^{[2,9]}$ Mericle et al. also reported good outcomes (50\%) even with poor clinical grades (HH 4 or higher) ${ }^{[9]}$ In our study, 24 of 39 patients $(61.5 \%)$ showed a favorable outcome, similar with other reports.

The PICA showed an anomalous pattern in $4-16 \%$ of cases and was classified into anterior medullary, lateral

Table 1: Baseline characteristics and summary of clinical and radiologic outcomes of ruptured posterior inferior cerebellar artery aneurysm according to the involved segment

\begin{tabular}{lccccccc}
\hline & \multicolumn{3}{c}{ Proximal PICA } & & \multicolumn{3}{c}{ Distal PICA } \\
\cline { 2 - 3 } \cline { 6 - 7 } & EVT & Surgery & $\boldsymbol{P}$ & & EVT & Surgery & $P$ \\
\hline No. of patient & 20 & 6 & & 7 & 6 & \\
Age, mean & 54.7 & 60.1 & 0.359 & & 63.7 & 58.1 & 1.000 \\
Initial HH & 2.33 & 2.22 & 0.344 & & 3.00 & 2.17 & 0.084 \\
Fisher Grade & 2.7 & 3.0 & 0.353 & 3.7 & 2.7 & 0.075 \\
Success rate & $100 \%$ & $100 \%$ & NA & & $87.5 \%$ & $100 \%$ & NA \\
Recurrence & $3(15 \%)$ & $0 \%$ & 1.000 & & $0 \%$ & $0 \%$ & NA \\
Complications & $4(20 \%)$ & $2(33.3 \%)$ & 0.596 & $3(42.9 \%)$ & $0 \%$ & 0.046 \\
Favorable outcome & $14(70 \%)$ & $2(33.3 \%)$ & 0.163 & $3(42.9 \%)$ & $5(83.3 \%)$ & 0.070 \\
Mean mRS & 1.75 & 3.50 & 0.152 & & 2.63 & 0.33 & 0.023 \\
Mean mRS except medical mortality * & 0.69 & 3.00 & 0.018 & 2.63 & 0.33 & 0.023 \\
\hline
\end{tabular}


Table 2: Summary of procedure related morbidity and mortality

\begin{tabular}{|c|c|c|c|c|c|c|c|c|}
\hline Case no & Sex/age & Involved segment & Treatment modality & Size (mm) & HH & Fisher & Complications & mRS (mo) \\
\hline 1 & $\mathrm{~F} / 73$ & Proximal & EVT & 16 & 3 & 3 & $\begin{array}{l}\text { PICA infarct } \\
\text { asymptomatic }\end{array}$ & $0(10)$ \\
\hline 2 & $\mathrm{~F} / 59$ & Proximal & $\mathrm{EVT}^{*}$ & 4 & 3 & 3 & $\begin{array}{l}\text { Unintended PAO } \\
\text { LCNP }\end{array}$ & $1(18)$ \\
\hline 3 & $\mathrm{M} / 48$ & Proximal & EVT & 3 & 2 & 2 & $\begin{array}{l}\text { Intraprocedural bleeding } \\
\text { Asymptomatic }\end{array}$ & $0(18)$ \\
\hline 4 & $\mathrm{~F} / 63$ & Proximal & EVT & 4 & 3 & 3 & Intraprocedural bleeding & $6(0)$ \\
\hline 5 & $\mathrm{~F} / 73$ & Proximal & EVT & 16 & 3 & 3 & Sequelae of SAH & $6(0)$ \\
\hline 6 & $F / 51$ & Proximal & EVT & 5 & 2 & 2 & Sepsis & $6(0)$ \\
\hline 7 & $\mathrm{~F} / 73$ & Proximal & EVT & 4 & 3 & 3 & ARDS & $6(0)$ \\
\hline 8 & $F / 58$ & Proximal & EVT & 4 & 4 & 3 & $\begin{array}{l}\text { Known CRF } \\
\text { pontine hemorrhage }\end{array}$ & $6(0)$ \\
\hline 9 & $\mathrm{~F} / 79$ & Proximal & surgery & 5 & 2 & 3 & $\begin{array}{l}\text { PICA infarct } \\
\text { LCNP }\end{array}$ & $4(1)$ \\
\hline 10 & $\mathrm{~F} / 69$ & Proximal & surgery & 3 & 3 & 3 & $\begin{array}{l}\text { PICA infarct } \\
\text { LNCP }\end{array}$ & $3(20)$ \\
\hline 11 & $\mathrm{~F} / 65$ & Proximal & surgery & 8 & 4 & 4 & $\mathrm{Ml}$ & $6(1)$ \\
\hline 12 & $\mathrm{M} / 59$ & Distal & $\mathrm{EVT}^{*}$ & 4 & 3 & 3 & $\begin{array}{l}\text { PICA infarct } \\
\text { LCNP }\end{array}$ & $4(72)$ \\
\hline 13 & $\mathrm{~F} / 66$ & Distal & $\mathrm{EVT}^{*}$ & 3 & 3 & 3 & $\begin{array}{l}\text { Intraprocedural bleeding } \\
\text { unintended PAO } \\
\text { Asymptomatic }\end{array}$ & $1(15)$ \\
\hline 14 & $\mathrm{~F} / 75$ & Distal & EVT * & 5 & 3 & 3 & Intraprocedural bleeding & $6(0)$ \\
\hline
\end{tabular}

medullary, tonsillomedullar, telovelotonsillar, and cortical segments according to the nearby structure along the course of the aneurysm. ${ }^{[4]}$ The first three segments are close to the brainstem and have multiple medullary perforators; however, beyond the tonsillomedullar segment, there were no critical perforators. Thus, decisions about proper treatment strategies, potential complications, and neurologic deficits can differ according to the location of the aneurysm.

\section{Surgery of posterior inferior cerebellar artery} aneurysms

The type of surgical approach, such as lateral suboccipital, midline subocciptial, and far lateral suboccipital, depends on the location of aneurysm. ${ }^{[4]}$ Microsurgical treatment can remove the blood clot, decompress increased intracranial pressure, and help checking the aneurysm and critical perforators around the aneurysm, as well as examine intracranial critical structure directly. ${ }^{[10]}$ It is also possible to control unexpected re-ruptures during the operation immediately. Orakciogle B et al. also reported on the decreased chance of additional procedures, such as extraventricular drainage (EVD) insertion or shunt by cerebrospinal fluid diversion, during surgery. ${ }^{[10]}$

The optimal strategy is to clip the aneurysm with the patent parent artery. The clip-wrap technique can be used to save the parent artery if the distinction of the neck and the parent artery is unclear. Surgical short-segment trapping of the parent artery, which can save more perforators through retrograde flow compared with parent artery occlusion (PAO) using glue injection, can be adjusted without any neurologic sequelae. We did not note any definite neurologic changes after microsurgical parent artery trapping in two of our patients. For ruptured distal PICA aneurysms, the mean initial HH for the microsurgical-treated groups and EVT was 2.17 and 3.00, respectively, and the rate of favorable outcome at surgery groups and EVT were $83.3 \%$ and $42.9 \%$ each. Also, in terms of mean mRS, microsurgical treatment showed favorable results ( 0.33 vs. $2.63, P=0.023)$.

But jugular tubercle, occipital condyle, and lower cranial nerves provide a narrow corridor; forced brain retraction to expose the neck of aneurysm in a narrow corridor can cause retraction injury and aggravate brain swelling during the acute phase. And the incidence of LCNP while manipulating the PICA is high in patients with a proximal PICA aneurysm through lateral and far-lateral suboccipital approaches. ${ }^{[1,4,12]}$ LCNP is also associated with the use of a temporary clip. ${ }^{[1]}$ Incidence of LCNP after microsurgical clip is about 10-45\%. Al-khayat $\mathrm{H}$ et al. reported $48.1 \%$ of LCNP after VA-PICA aneurysm clipping. ${ }^{[1]}$ Fortunately, most LCNP resolve and the overall results are favorable in up to $76 \%$ of cases approximately 3 to 6 months after the procedure. ${ }^{[1,12]}$ 
In our study, two of six patients (33.3\%) that received microsurgical treatment at the proximal PICA aneurysm showed LCNP, and all patients progressed to develop permanent moderate neurologic deficits. However, there were no complications with unfavorable outcomes in the distal PICA aneurysm after microsurgical treatment. This is because surgical exposure of distal PICA aneurysm is not difficult and does not have the need to manipulate lower cranial nerve. Also, there are no critical perforators at distal PICA.

Endovascular treatment of posterior inferior cerebellar artery aneurysms

Recently, EVT has become the primary option in the treatment of aneurysms. EVT decreases the risk of direct brainstem injury and reduces the burden of anesthesia in patients with a very poor clinical status or with a poor past medical history. Ryttlefors et al. reported that the survival rate without disability in the EVT group is significantly higher in subarachnoid hemorrhage (SAH) cases compared with surgical treatment. ${ }^{[5]}$ Advanced techniques and the development of supporting devices can cause a complex aneurysm to be occluded completely. Consequently, there are many published reports regarding the safety and feasibility of EVT in the treatment of a PICA aneurysm. ${ }^{[7]}$ In patients with proximal PICA aneurysms, EVT showed more favorable outcome than surgery groups in our study $(70 \%$ vs $33.3 \%$, $P=0.163)$. Also, there was no statistically significant differences between the EVT and surgery group in terms of mean mRS, though the mean mRS in the EVT group was lower at 1.75 versus 3.5 in the surgery group. In cases of poor medical history, poor initial clinical status, and higher risk of general anesthesia, we performed EVT instead of surgery. In the EVT group of proximal PICA aneurysms, four patients died due to procedure-unrelated problems such as sepsis, acute respiratory distress syndrome, stem hemorrhage, and sequelae of SAH. Thus, if patients with deaths unrelated to the procedure were excluded, difference of mean mRS between two treatment modalities of proximal PICA aneurysms was statistically significant $(0.69$ vs $3.00, P=0.018)$.

Nevertheless, there are also many reports regarding the limitations of EVT, especially with respect to a distal PICA. ${ }^{[3,5]}$ The small diameter and tortuous nature of the PICA vessel makes interventions difficult, as one must navigate into the aneurysm through 1 or 2 loops (cranial and caudal loop) without vessel dissection or vasospasm while stably guiding the catheter. Aneurysmal rupture is especially associated with the procedure such as jumping of the microcatheter, and about 11-19\% of cases resulting in a catastrophic outcome. ${ }^{[1]}$ Procedural rupture developed in four patients (two proximal and two distal PICA aneurysms). One improved without any resulting neurologic deficits, one had mild neurologic deficit; however, two patients died.
Asymptomatic or symptomatic embolic infarct and unexpected parent artery occlusion, procedure-related thrombus formation can occur during EVT. Occasional LCNP due to brainstem infarct has been reported. ${ }^{[5]}$ Fortunately, brainstem infarct after parent artery occlusion of the proximal segment is low, because the PICA territory is also supplied via the contralateral PICA, ipsilateral anterior inferior cerebellar artery, or superior cerebellar artery in many cases and the pathologic segment may be associated with the absence of a normal perforating branch. ${ }^{[3]}$ Plexiform networks also develop on the medullary surface, and in some cases, the medulla is exclusively supplied by the anterior spinal artery and vertebral artery branches rather than the PICA. ${ }^{[3]}$ In our study, there were three cases of PICA infarct (two proximal and one distal PICA aneurysm); one was due to parent artery thrombus, another developed in a very large aneurysm during coiling embolization of proximal PICA aneurysm, the other patient of distal PICA aneurysm had a permanent moderate neurologic deficit because of glue migration to proximal segment. In the case of distal PICA aneurysm with EVT, three of seven patients (42.9\%) developed complications; although the results were statistically insignificant $(P=0.462)$, the complication rate of EVT was higher than that of surgery of distal PICA aneurysms. Additionally, recanalization after treatment and the need for a follow-up image evaluation are other drawbacks of EVT.

\section{Other treatment methods}

Another treatment method is endovascular or microsurgical VA occlusion. This reduces blood flow at both the PICA and the aneurysm without blocking blood flow to critical perforators and cortical branches, and it allows for collateral vessels to develop; complication rates after sacrificing the PICA are thus decreased. ${ }^{[13]}$ One problem is it takes a long time to create a thrombosis in the aneurysm sac, and the chance of aneurysmal rupture exists until the thrombus is completely formed. ${ }^{[13]}$ Bypass surgery is one of the fine treatment modalities, but there is no evidence to fully support that a bypass surgery performance yields a better outcome in unstable SAH patients with ruptured PICA aneurysm. Bypass technique could rather prolong the operation time and considering its possible risk for consequent procedure-related complication. Neurosurgeons must also take caution in certain cases, since the failure rate of bypass is high in cases with a ruptured aneurysm. ${ }^{[6]}$

\section{Study limitations}

This study suffers from the usual limitations that arise from retrospective data reviews. The small number of patients in the subgroups and inherent biases involved in patient selection for treatment preclude making any definitive recommendations. Nevertheless, the results provide some guidance to the clinician when considering 
EVT or surgery for the treatment of patients with ruptured PICA aneurysms.

\section{CONCLUSIONS}

Both surgery and EVT are feasible treatment modalities for preventing rebleeding in the treatment of proximal or distal PICA aneurysms. EVT appears to result in a more favorable clinical outcome in patients with proximal PICA aneurysms, while surgery results in a more favorable outcome in patients with distal PICA aneurysms. Additional experience and follow-up are needed in a larger series to state the efficacy of this strategy.

\section{Financial support and sponsorship}

Nil.

\section{Conflicts of interest}

There are no conflicts of interest.

\section{REFERENCES}

I. Al-khayat H, Al-Khayat H, Beshay J, Manner D, White J. Vertebral artery-posteroinferior cerebellar artery aneurysms: Clinical and lower cranial nerve outcomes in 52 patients. Neurosurgery 2005;56:2-10.

2. Bacigaluppi S, Bergui M, Crobeddu E, Garbossa D, Ducati A, Fontanella M. Aneurysms of the medullary segments of the posterior-inferior cerebellar artery: Considerations on treatment strategy and clinical outcome. Neurol Sci 2013;34:529-36.
3. Chalouhi N, Jabbour P, Starke RM, Tjoumakaris SI, Gonzalez LF, Witte S, et al. Endovascular treatment of proximal and distal posterior inferior cerebellar artery aneurysms. J Neurosurg 2013;1 18:991-9.

4. Hudgins RJ, Day AL, Quisling RG, Rhoton AL, Jr., Sypert GW, Garcia-Bengochea F. Aneurysms of the posterior inferior cerebellar artery. A clinical and anatomical analysis. J Neurosurg 1983;58:381-7.

5. Isokangas JM, Siniluoto T, Tikkakoski T, Kumpulainen T. Endovascular treatment of peripheral aneurysms of the posterior inferior cerebellar artery. AJNR Am J Neuroradiol 2008;29:1783-8.

6. Korja M, Sen C, Langer D. Operative nuances of side-to-side in situ posterior inferior cerebellar artery-posterior inferior cerebellar artery bypass procedure. Neurosurgery 2010;67:47I-7.

7. Lewis SB, Chang DJ, Peace DA, Lafrentz PJ, Day AL. Distal posterior inferior cerebellar artery aneurysms: Clinical features and management. J Neurosurg 2002;97:756-66.

8. Lister JR, Rhoton AL, Jr., Matsushima T, Peace DA. Microsurgical anatomy of the posterior inferior cerebellar artery. Neurosurgery 1982;10:170-99.

9. Mericle RA, Reig AS, Burry MV, Eskioglu E, Firment CS, Santra S. Endovascular surgery for proximal posterior inferior cerebellar artery aneurysms: An analysis of Glasgow Outcome Score by Hunt-Hess grades. Neurosurgery 2006;58:619-25.

10. Orakcioglu B, Schuknecht B, Otani N, Khan N, Imhof HG, Yonekawa Y. Distal posterior inferior cerebellar artery aneurysms: Clinical characteristics and surgical management. Acta Neurochir 2005; 147:1 I3I-9.

II. Peluso JP, van Rooij WJ, Sluzewski M, Beute GN, Majoie CB. Posterior inferior cerebellar artery aneurysms: Incidence, clinical presentation, and outcome of endovascular treatment. AJNR Am J Neuroradiol 2008;29:86-90.

12. Singh RK, Behari S, Kumar V, Jaiswal AK, Jain VK. Posterior inferior cerebellar artery aneurysms: Anatomical variations and surgical strategies. Asian J Neurosurg 2012;7:2-1I.

13. Song HH, Won YD, Kim YJ, Kim BS. The endovascular management of saccular posterior inferior cerebellar artery aneurysms. Korean J Radiol 2008;9:396-400. 